\title{
MÉTODOS ESTATÍSTICOS DE SUAVIZAÇÃO EXPONENCIAL HOLT- WINTERS PARA PREVISÃO DE DEMANDA EM UMA EMPRESA DO SETOR METAL MECÂNICO
}

\section{STATISTICAL METHODS EXPONENTIAL SMOOTHING HOLT- WINTERS TO FORECAST DEMAND IN A COMPANY OF THE METAL MECHANIC SECTOR}

\author{
Andrey Jonas Veríssimo ${ }^{1}$; Custodio da Cunha Alves ${ }^{2}$; Elisa Henning ${ }^{3}$; Claiton Emílio do Amaral ${ }^{4}$; \\ Altair Carlos da Cruz ${ }^{5}$ \\ ${ }^{1}$ Universidade da Região de Joinville - UNIVILLE - Santa Catarina - Brasil \\ andrey-verissimo@bol.com.br \\ ${ }^{2}$ Universidade da Região de Joinville - UNIVILLE - Santa Catarina - Brasil \\ custodio.alves@univille.br \\ ${ }^{3}$ Universidade do Estado de Santa Catarina - UDESC - Santa Catarina - Brasil \\ elisa.henning@udesc.br \\ ${ }^{4}$ Universidade da Região de Joinville - UNIVILLE - Santa Catarina - Brasil \\ claiton.emilio@gmail.com \\ ${ }^{5}$ Universidade da Região de Joinville - UNIVILLE - Santa Catarina - Brasil \\ altair@univille.edu.br
}

\begin{abstract}
Resumo
Os métodos estatísticos de suavização exponencial Holt-Winters são amplamente utilizados para fornecer previsões de curto prazo para os dados de vendas e os níveis de demanda, devido a sua simplicidade, baixo custo de operação, boa precisão, capacidade de ajustamento automático e rápido a mudanças na série temporal em análise. O objetivo principal desse trabalho é a aplicação desses métodos estatísticos para estabelecer em curto prazo previsões precisas para o planejamento de demanda de vendas e a partir dessa análise de previsão de demanda tomar decisões que contemple a busca contínua da gestão otimizada do processo produtivo de uma empresa do setor metal mecânico, localizada na região norte do Estado de Santa Catarina. Para esta aplicação propõe-se uma análise de séries temporais da venda de duas categorias de produtos com efeitos sazonais e que têm impacto significativo nos custos e investimentos dessa empresa. Na escolha do método de projeção para cada uma dessas séries é considerado os tipos efeitos sazonais que estabelecem a utilização adequada entre os métodos Holt-Winters Sazonal Multiplicativo e o Sazonal Aditivo. Os resultados obtidos, com o delineamento deste estudo baseado exclusivamente em dados históricos da demanda de vendas foram fundamentais para o analista de previsão desta empresa, destinar especial atenção ao grau de acurácia dos métodos preditivos utilizados e na adoção de um sistema de monitoramento dos erros de previsão, aspecto importante para tomada de ações corretivas, quando necessário.
\end{abstract}

Palavras-chave: Métodos Estatísticos; Séries Temporais; Previsão de Demanda. 


\section{Introdução}

A previsão de demanda constitui-se em uma das atividades de gestão mais importantes para a tomada de decisões gerenciais, uma vez que o sucesso futuro de qualquer empresa depende muito de como o gestor é mais experiente em detectar tendências e desenvolver estratégias adequadas. Neste contexto, o planejamento e o direcionamento estratégico das empresas dependem da identificação e da previsão correta das mudanças emergentes no ambiente de negócios.

A pesquisa por métodos estatísticos de previsão adequados para o prognóstico quantitativo de uma variável, e como medir a qualidade dessa previsão tem sido um diferencial cada vez mais utilizado pelas empresas para antecipar cenários futuros. Assim é possível planejar, alocar e dimensionar recursos de modo a tentar reduzir custos desnecessários com decisões equivocadas. As empresas recorrem frequentemente a esses métodos estatísticos em áreas diversas como a gestão de inventário, planejamento da produção, escalonamento dos recursos humanos, controle do processo, entre outras. O objetivo central da utilização destes métodos é prever os acontecimentos futuros, tendo como propósito a redução do risco na tomada de decisão.

Este trabalho tem por objetivo principal a aplicação do método estatístico de suavização exponencial por séries temporais Holt-Winters para estabelecer a um curto prazo previsões precisas para o planejamento de demanda de vendas. E, a partir dessa análise de previsão de demanda tomar decisões que contemplem a busca contínua da gestão otimizada do processo produtivo de uma empresa do setor metal mecânico, localizada na região norte de Santa Catarina.

Este artigo está estruturado em cinco seções, incluindo a presente introdução. A seção 2 aborda a previsão de demanda como ferramenta de apoio à logística. Um breve referencial teórico sobre métodos estatísticos de previsão com destaque aos métodos de séries temporais é apresentado na seção 3. A seção 4 apresenta os principais métodos estatísticos de suavização exponencial atribuindo maior ênfase aos métodos de previsão de demanda aplicados neste trabalho, cujas séries temporais exibem tanto tendência quanto sazonalidade. A seção 5 mostra a aplicação do método Holt-Winters selecionado como melhor método de previsão de demanda e a otimização dessa previsão com modelagem de planilhas eletrônicas em ambiente MS-Excel® e, finalmente, a seção 6 apresenta as considerações finais.

\section{Previsão de demanda}

A previsão consiste na estimação e análise da demanda futura para um produto em particular, componente ou serviço, utilizando entradas como relações históricas de vendas, estimação de marketing e informação promocional através de diferentes técnicas de previsão. Segundo Bermúdez, Segura e Vercher (2006) e Krajewski, Ritzman e Malhotra (2007), a base para 
a maioria das decisões orientadas para o futuro das empresas é estabelecida pela previsão de demanda, logo, ela é uma ferramenta essencial para decisões de gestão e de planejamento estratégico.

O processo de estabelecer previsão constitui-se em uma das mais importantes ações no que se refere a diversas tomadas de decisões no dia a dia de uma organização. No setor industrial, por exemplo, a mensuração da qualidade da previsão é fundamental para reduzir de forma significativa a variabilidade de um processo produtivo uma vez que produtos devem ser fabricados dentro das medidas de conformidade. Isso significa tanto as médias ficarem no alvo, quanto a dispersão das medições também contidas em valores mínimos (SOUZA; SAMOHYL; MIRANDA, 2008). Este processo é uma série de atividades relacionadas que transformam uma ou mais entradas em uma ou mais saídas. Todas as atividades de trabalho são realizadas em processos, e previsão não é exceção. Estas atividades são mostradas na Figura 1.

Figura 1- Processo de previsão

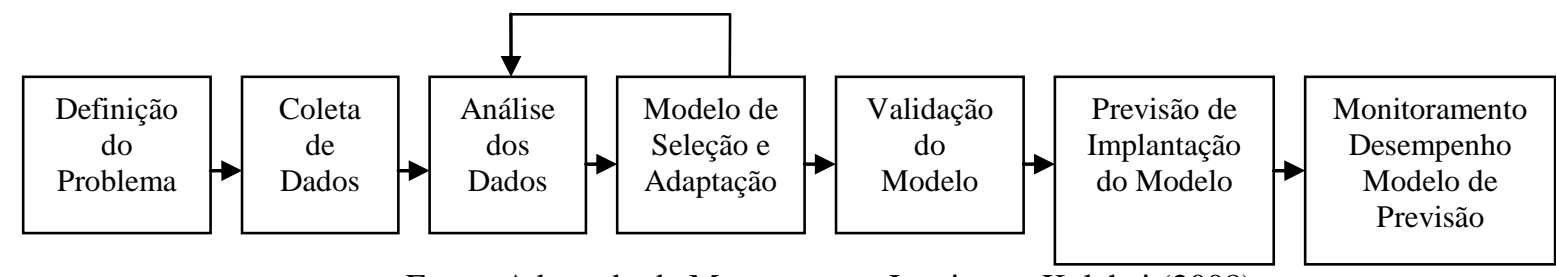

Fonte: Adaptado de Montgomery, Jennings e Kulahci (2008)

O fornecimento de previsões ou projeções de demanda constitui atualmente, uma parte fundamental da logística pelas implicações que uma mudança na estratégia decorrente dos principais processos da cadeia de abastecimento (gestão de estoque, abastecimento, transporte, fabricação, nível de serviço, etc.) e para os benefícios que proporcionam sua correta estimativa e monitoramento.

\section{Métodos Estatísticos de Previsão}

Os métodos estatísticos de previsão são ferramentas fundamentais para projetar comportamentos futuros, tendo como propósito a redução do risco na tomada de decisão. Normalmente, as previsões apresentam erros, mas a magnitude dos seus erros depende muito do sistema de previsão utilizado. Um dos maiores problemas associados à utilização das previsões de demanda no apoio à tomada de decisões é a escolha adequada do método de previsão a ser implementado. Diante disso, uma maior disponibilização de recursos no método de previsão escolhido, permite melhorar a precisão da previsão e, assim, eliminar alguns dos prejuízos resultantes da incerteza existente no processo de tomada de decisão.

As previsões de demanda são elaboradas a partir de métodos estatísticos de previsão 
classificados, basicamente, em duas categorias: qualitativos e quantitativos. Os métodos qualitativos baseiam-se em opiniões subjetivas, especulações e intuição de especialistas. Os métodos quantitativos servem-se da manipulação matemática de dados históricos (quantificados) para projetar no futuro padrões de comportamento identificados por esses dados históricos. Estes métodos partem do pressuposto que o padrão da série histórica terá continuidade no futuro e são subdivididos em dois tipos, conforme envolvam séries causais ou séries temporais. Os métodos causais envolvem a determinação de fatores que se relacionam com a variável a ser prevista enquanto que os métodos de previsão de séries temporais envolvem a projeção de valores futuros de uma variável, com base, inteiramente, em observações do presente e do passado dessa variável.

\subsection{Métodos Estatísticos de Previsão por Séries Temporais}

Os métodos estatísticos de previsão por séries temporais baseiam-se na idéia de que as observações passadas da série contêm informações sobre o seu padrão de comportamento no futuro. A essência desses métodos consiste em identificar o padrão da série, separando-o do ruído contido nas observações individuais, e utilizá-lo para prever os valores futuros da série (HENNING, ALVES e KONRATH, 2010). O objetivo da análise de séries temporais é encontrar modelos (estatísticos e/ou matemáticos) que permitem descrever de forma adequada o processo de geração de dados para prever o comportamento futuro das variáveis para um determinado horizonte de planejamento.

Apesar de a maior parte dos métodos de previsão de séries temporais estarem fundamentada apenas na análise das observações da série de interesse para a especificação de algum modelo que descreva essas observações, alguns procedimentos de previsão tentam explicar o comportamento de uma serie temporal pela evolução dos fenômenos observáveis de outras séries.

A previsão de demanda utilizando métodos quantitativos pode ser desenvolvida através de vários métodos estatísticos. O emprego de cada método depende basicamente do comportamento da série temporal que se deseja analisar. Uma série temporal pode exibir até quatro características diferentes em seu comportamento: média, sazonalidade, ciclo e tendência (MAKRIDAKIS, WHEELWRIGHT e HYNDMAN, 1998). A característica sazonal da série é quando padrões cíclicos de variação se repetem em intervalos relativamente constantes de tempo. A característica cíclica existe quando a série exibe variações ascendentes e descendentes, porém, em intervalos não regulares de tempo. Finalmente, a característica de tendência ocorre quando a série apresenta comportamento ascendente ou descendente por um período de tempo (PELLEGRINI e FOGLIATTO, 2001).

Uma vez que muitos métodos estatísticos de previsão estão à disposição para a modelagem de dados de séries temporais, cada qual com suas capacidades e limitações, é impossível saber de 
antemão qual método será mais eficiente para determinado conjunto de dados. Assim, uma abordagem comum para análise de série temporal segundo Ragsdale (2009), envolve o teste de várias técnicas de modelagem em um dado conjunto de dados e a avaliação de como essas técnicas explicam o comportamento passado da variável de série temporal. Essas técnicas podem ser avaliadas através de gráficos de linha que ilustram os reais dados versus os valores previstos pelas diversas técnicas de modelagem.

A seleção do método estatístico e previsão de séries temporais adequado dependem de vários fatores, tais como o comportamento do fenômeno observável ou o conhecimento a priori que se tenha sobre a sua natureza e do objetivo da análise. As medidas quantitativas mais formais de desempenho são em muitos casos de previsão, o principal critério de seleção de um método de previsão. Estas permitem a medição da eficácia de um método de previsão, mostrando o quanto ele e capaz de reproduzir os dados que são já conhecidos. O diferencial para seleção de um método previsão está na precisão da previsão futura, ou seja, na capacidade do modelo gerar previsões que sejam tão precisas quanto possível.

Assim, a escolha do método de previsão mais apropriado pode ser realizada a partir de uma ou mais medidas estatísticas de desempenho sobre erros de previsão, baseadas na somatória dos erros gerados por cada método $\left(e_{t}=Z_{t}-\hat{Z}_{t}\right)$, onde $Z_{t}$ é o valor observado atual (valor real) para um

período de tempo t, e $\hat{Z}_{t}$ é o valor previsto para esse mesmo período de tempo. A avaliação e a comparação entre os métodos de previsão são realizadas através destas estatísticas que medem o grau de ajustamento do modelo aos dados. Dentre estas estatísticas uma das medidas mais utilizadas é erro quadrático médio (MSE - Mean Squared Error) conforme equação (1). Esta medida apresenta vantagem de ser a mais facilmente manipulável matematicamente.

$$
M S E=\frac{1}{n} \sum_{t=1}^{n} e_{t}^{2}
$$

Os métodos estatísticos de série temporais incluem os seguintes modelos de previsão: Médias Móveis, Box-Jenkins e o de Suavização Exponencial, objeto de estudo desse trabalho.

\section{Métodos de Suavização Exponencial}

A suavização ou ajuste exponencial é também um método estatístico que parte de uma equação de médias móveis, ponderadas exponencialmente, com o objetivo de produzir ajustes nas variações aleatórias dos dados de determinada série temporal. Esse procedimento de suavização utiliza uma ponderação distinta para cada valor observado na série temporal, de modo que valores mais recentes recebam pesos maiores. Assim, os pesos formam um conjunto que decai exponencialmente a partir de valores mais recentes (GARDNER, 2006). 
Os métodos de previsão por suavização exponencial se utilizam apenas de observações da própria série de dados, não dependendo de nenhuma variável externa para realizar previsões. (SOUZA, SAMOHYL e MIRANDA, 2009). Esses métodos, segundo Gardner (1985; 2006), são reconhecidos como uma das ferramentas mais empregadas na previsão de curto prazo de séries temporais. Sua popularidade se deve principalmente a sua simples formulação, facilidade de ajustes e boa precisão (MAKRIDAKIS e HIBON, 2000). Os procedimentos destes métodos incorporam de maneira simples e compreensível o nível, a tendência e a sazonalidade que apresentam os dados da série temporal, assim como o erro de previsão, que é o componente estocástico do método (HYNDMAN et al, 2002; 2008).

Segundo Morettin e Toloi (2006), a classificação de um método suavização exponencial além da componente tendência pode também estar relacionada com o fator sazonalidade e pela inclusão do erro, tanto na forma aditiva como na multiplicativa. A seguir são apresentadas as três variações básicas de suavização exponencial comumente utilizada na prática.

\subsection{Método de Suavização Exponencial Simples}

O tipo mais simples de série temporal é aquele em que os valores da série flutuam aleatoriamente em torno de um valor fixo, sem apresentar qualquer tendência. Se a série temporal mantém-se constante sobre um nível médio, uma suavização exponencial simples proposta por Brown $(1959,1963)$ pode ser usada para a previsão de valores futuros da série. Sua representação matemática vem dada por (MAKRIDAKIS, WHEELWRIGHT e HYNDMAN, 1998).

$$
\hat{Z}_{t+1}=\alpha Z_{t}+(1-\alpha) \hat{Z}_{t}, \quad 0 \leq \alpha \leq 1
$$

onde $\hat{Z}_{t+1}$ é o valor previsto para o período de tempo $t+1$, $\alpha$ é o parâmetro constante de suavização do método; que pode assumir qualquer valor entre 0 e $1(0 \leq \alpha \leq 1)$; $Z_{t}$ é o valor observado atual (valor real) para o período de tempo $t$ e, $\hat{Z}_{t}$ é o valor previsto para o mesmo período de tempo $t$. De acordo com a equação (2), o valor previsto para o próximo período $\hat{Z}_{t+1}$ é uma combinação do valor previsto atual $\hat{Z}_{t}$ e o valor observado atual $Z_{t}$. O valor da constante de suavização $\alpha$ é arbitrário, sendo que a determinação de seu melhor valor pode ser realizada iterativamente, utilizando alguma forma de comparação, como, por exemplo, o erro quadrático médio (MSE). Desta forma, selecionase aleatoriamente um valor inicial para a constante, a partir do qual previsões são geradas. Comparam-se os valores previstos com os reais, e calcula-se a média do quadrado das diferenças entre os mesmos; o parâmetro que minimiza essa média é utilizado no modelo final (PELLEGRINI e FOGLIATTO, 2001). 


\subsection{Método de Holt (Suavização Exponencial Dupla)}

A suavização exponencial dupla, também conhecida como método de Holt é muitas vezes uma ferramenta eficaz de previsão desenvolvida por Holt (1957) que expandiu a suavização exponencial simples para dados de série temporal que exibem uma tendência linear. Este método oferece refinamentos adicionais na modelagem, à medida que introduz uma constante de suavização que afeta a tendência da série. A função de previsão do método de Holt é representada por

$$
\hat{Z}_{t+n}=L_{t}+n T_{t}
$$

onde a previsão para o período de tempo $t+n\left(\hat{Z}_{t+n}\right)$ é igual estimativa do nível esperado da série temporal no período de tempo t $\left(L_{t}\right)$ mais a influência esperada da tendência (taxa de aumento ou redução) durante os próximos $n$ períodos ( $n T$ ). A função de previsão na equação (3) pode ser utilizada para obter períodos de tempo $n$ para previsões no futuro, onde $n=1,2,3$, e assim por diante.

O método desenvolvido por Holt recorre ao uso dos parâmetros $\alpha$ e $\beta$ denominadas constantes de suavização. Nesse método, além da função (3) que calcula a previsão duas outras funções são utilizadas para estimar o nível e a tendência da série temporal, conforme equações (4) e (5), respectivamente.

$$
\begin{array}{ll}
L_{t}=\alpha Z_{t}+(1-\alpha)\left(L_{t-1}+T_{t-1}\right), & 0 \leq \alpha \leq 1 \\
T_{t}=\beta\left(L_{t}-L_{t-1}\right)+(1-\beta) T_{t-1}, & 0 \leq \beta \leq 1
\end{array}
$$

onde $L_{t}$ é a estimação para o nível da série no período $t$ e $T_{t}$ é a estimação da inclinação (tendência) da série no mesmo período $t$. A equação (3) ajusta diretamente $L_{t}$ para a tendência do período anterior $T_{t-1}$, adicionando o último valor suavizado $L_{t-1}$.

Os valores de $\alpha$ e $\beta$ são encontrados por ensaio sobre a série histórica, sendo frequente selecionar aqueles que minimizam o erro quadrático médio. Para enfrentar o problema da subjetividade na seleção dos parâmetros $\alpha$ e $\beta$ algumas investigações (BILLAH et al, 2006; GELPER, FRIED e CROUX, 2010) desenvolvidas que mostram como selecionar esses parâmetros otimizando alguma das medidas de desempenho dos prognósticos, como erro médio (ME), erro absoluto médio (MAE) e erro quadrático médio (MSE). Enquanto isso, Rasmussen (2004), propõe a obtenção tanto dos parâmetros $\alpha$ e $\beta$ que otimizam o método de previsão quanto os valores iniciais, definindo para tal o MSE como função objetivo para minimizar e resolver o método, com auxílio da ferramenta solver do MS-Excel. 


\subsection{Método de Holt-Winters (Suavização Exponencial Tripla)}

Os métodos estatísticos de séries temporais são técnicas quantitativas frequentemente utilizadas para realizar prognósticos de variáveis, dentre os quais se encontram os métodos de suavização exponencial, em particular o método Holt-Winters que é uma expansão do método Holt (1957) desenvolvida por Winter (1960) para aplicar em séries temporais que exibem tendência e sazonalidade. É um dos métodos mais utilizados para previsão de curto prazo da demanda, devido a sua simplicidade, baixo custo de operação, boa precisão e capacidade de ajustamento automático e rápido a mudanças na série em análise. Partindo do princípio que determinadas séries possuem um fator sazonal, além do nível e tendência que capta características da série que se repetem a intervalos regulares de tempo, Winter propõe métodos de projeção para essas séries, considerando dois tipos de efeitos sazonais: multiplicativo e aditivo.

\subsubsection{Método de Holt-Winter para Efeitos Sazonais Multiplicativos}

O método de Holt-Winter para efeitos sazonais multiplicativos é utilizado na modelagem de dados onde a amplitude do ciclo sazonal varia proporcionalmente ao nível da série com o passar do tempo. Seja um modelo de série sazonal, de período s, fator sazonal multiplicativo e tendência aditiva, isto é,

$$
Z_{t}=L_{t} S_{t}+T_{t}+\in_{t}, \quad t=1,2 \ldots
$$

que descreve o comportamento estrutural da série. As projeções dos valores futuros da série são efetuadas através da função de previsão do método representada por

$$
\hat{Z}_{t+n}=\left(L_{t}+n T_{t}\right) S_{t-s+n}, \quad n=1,2 \ldots
$$

onde $\hat{Z}_{t+n}$ é a previsão para n períodos à frente $(t+n)$. Nesse método, além da função (7) que calcula a previsão três outras funções são utilizadas para estimar o nível, a tendência da série no período atual e os valores do fator sazonal correspondente ao último período de sazonalidade, conforme equações (8), (9) e (10), respectivamente.

$$
\begin{array}{lr}
L_{t}=\alpha \frac{Z_{t}}{S_{t-s}}+(1-\alpha)\left(L_{t-1}+T_{t-1}\right) ; & 0 \leq \alpha \leq 1 \\
T_{t}=\beta\left(L_{t}-L_{t-1}\right)+(1-\beta) T_{t-1} ; & 0 \leq \beta \leq 1 \\
S_{t}=\gamma \frac{Z_{t}}{L_{t}}+(1-\gamma) S_{t-s}, & 0 \leq \gamma \leq 1
\end{array}
$$


onde $\alpha, \beta$ e $\gamma$ são as constantes de suavização que controlam o peso relativo ao nível ( $\left.L_{t}\right)$, a tendência $\left(T_{t}\right)$ e a sazonalidade $\left(S_{t}\right)$, respectivamente. As equações (8) e (10) assumem que, no período de tempo $t$, existe uma estimativa do índice sazonal no período de tempo $t$-s ou que existe um valor $S_{t-s}$. Assim, precisamos estimar os valores para $S_{1}, S_{2}, \ldots \ldots, S_{p}$. Uma forma simples de fazer estas estimativas sazonais iniciais necessárias para a utilização das equações de suavização é permitir que

$$
S_{t}=\frac{Z_{t}}{\sum_{n=1}^{s} \frac{Z_{n}}{s}}, t=1,2, \ldots, s .
$$

\subsubsection{Método de Holt-Winter para Efeitos Sazonais Aditivos}

O método de Holt-Winter para efeitos sazonais aditivos é utilizado na modelagem de dados onde a amplitude do ciclo sazonal independe do nível local da série, ou seja, permanece constante com o passar do tempo. Seja um modelo cuja série sazonal, de período s, é formada pela soma do nível, tendência, um fator sazonal e um erro aleatório, dado por

$$
Z_{t}=L_{t}+T_{t}+S_{t}+\epsilon_{t} . \quad t=1,2 \ldots
$$

As projeções dos valores futuros da série são efetuadas através da função de previsão do método representada por

$$
\hat{Z}_{t+n}=L_{t}+n T_{t}+S_{t-s+n}, \quad n=1,2 \ldots
$$

onde $\hat{Z}_{t+n}$ é a previsão para $n$ períodos à frente $(t+n)$. Nesse método, além da função (13) que calcula a previsão, três outras funções são utilizadas para estimar o nível, a tendência da série no período atual e, os valores do fator sazonal correspondente ao último período de sazonalidade, conforme equações (14),(15) e (16) respectivamente,

$$
\begin{array}{ll}
L_{t}=\alpha\left(Z_{t}-S_{t-s}\right)+(1-\alpha)\left(L_{t-1}+T_{t-1}\right) ; & 0<\alpha<1 \\
T_{t}=\beta\left(L_{t}-L_{t-1}\right)+(1-\beta) T_{t-1} ; & 0<\beta<1 \\
S_{t}=\gamma\left(Z_{t}-L_{t}\right)+(1-\gamma) S_{t-s}, & 0<\gamma<1
\end{array}
$$

onde $\alpha, \beta$ e $\gamma$ são as constantes de suavização que controlam o peso relativo ao nível $\left(L_{t}\right)$, a tendência $\left(T_{t}\right)$ e a sazonalidade $\left(S_{t}\right)$, respectivamente. As equações (14) e (16) assumem que, no período de tempo $t$, existe uma estimativa do índice sazonal no período de tempo $t$-s ou que existe 
um valor $S_{t-s}$. Assim, precisamos estimar os valores para $S_{1}, S_{2}, \ldots \ldots, S_{p}$. Uma forma simples de fazer estas estimativas sazonais iniciais necessárias para a utilização das equações de suavização é permitir que

$$
S_{t}=Z_{t}-\sum_{n=1}^{s} \frac{Z_{n}}{s}, \quad \quad 1,2, \ldots, s
$$

\section{Aplicação}

Neste trabalho para determinar as previsões de demanda em um horizonte de quatro trimestres de 2012, são empregados modelos estatísticos de suavização exponencial de HoltWinters com auxílio de planilhas. Para tal aplicação são utilizados dados reais referentes ao histórico de vendas de uma indústria do setor metal mecânico do norte de Santa Catarina, cujos dados revelam tanto características de tendência quanto de sazonalidade.

As séries temporais referentes às vendas trimestrais de duas categorias de produtos $\mathrm{A}$ e $\mathrm{B}$ dessa indústria correspondem aos dados técnicos relevantes e relativos às características técnicas de montagem de equipamentos, encontrados no contexto real durante o período de 2007 a 2011 . De posse, de todas essas características obtidas a partir do histórico de montagem de equipamentos num período de cinco anos, a próxima etapa é a classificação dos dados para o estudo das séries temporais envolvidas neste estudo. A análise de séries temporais utilizadas nesse trabalho envolve as demandas desses dois produtos A e B distribuídos em duas linhas de montagem de equipamentos W e R agrupados por tamanho, cuja configuração que caracteriza esses produtos é o fato de se apresentarem na forma modular. Durante esses cinco anos de operação essa indústria obteve um determinado crescimento na venda desses produtos conforme os gráficos da Figura 1.

Figura 1 - Dados históricos de vendas (em milhares) para a previsão de demanda dos produtos A e B
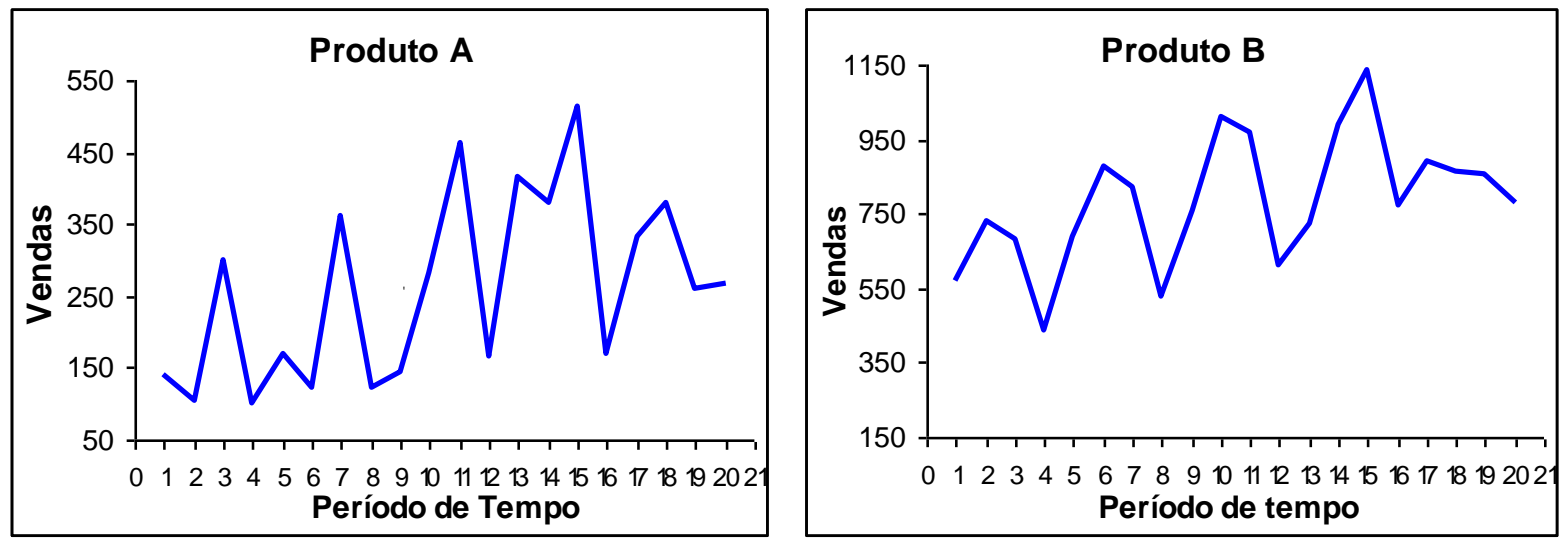

Fonte: Autoria própria (2012)

Como se pode observar, ambas as séries temporais não estacionárias ilustram uma determinada tendência crescente nas vendas e exibem a presença de efeitos sazonais ao longo desse 
período. Esta análise indica uma possível aplicação de métodos de suavização exponencial que apresentem tais características. Além da análise gráfica para prever o valor dessa variável de série temporal, realizou-se um estudo das medidas de desempenho e otimização dos métodos de previsão como, por exemplo, o MSE e as constantes de suavização exponencial que minimizam MSE no método de previsão.

A partir da modelagem e análise dessas séries temporais foi realizado um estudo preliminar envolvendo os diferentes métodos de suavização exponencial para a previsão de venda e fabricação dos trimestres de 2012 de cada uma das duas categorias de produtos. Nesse estudo, avaliou-se a capacidade preditiva dos diferentes métodos de suavização exponencial por série 1 , sob a premissa de conhecer os dados reais do ano de previsão. O Quadro 1 mostra um resumo do desempenho relativo aos diferentes métodos de suavização exponencial desse estudo, obtido a partir do valor do erro quadrático médio (MSE).

Quadro 1 - Comparativo entre a estatística de desempenho MSE para os métodos de suavização exponencial

\begin{tabular}{|c|c|c|c|c|}
\hline & \multicolumn{2}{|r|}{ PRODUTO A } & \multicolumn{2}{|c|}{ PRODUTO B } \\
\hline Método de Previsão & MSE & $\begin{array}{l}\text { Constante de } \\
\text { Suavização }\end{array}$ & MSE & $\begin{array}{l}\text { Constante de } \\
\text { Suavização }\end{array}$ \\
\hline Exponencial Simples & 18613 & $\alpha=0,5$ & 33759 & $\alpha=0,5$ \\
\hline $\begin{array}{l}\text { Exponencial Simples } \\
\text { Otimizado }\end{array}$ & 16354 & $\alpha=0,25$ & 30924 & $\alpha=0,27$ \\
\hline Holt & 22890 & $\alpha=0,5$ e $\beta=0,5$ & 45079 & $\alpha=0,5$ e $\beta=0,5$ \\
\hline $\begin{array}{l}\text { Holt } \\
\text { Otimizado }\end{array}$ & 16171 & $\alpha=0,20$ e $\beta=0,07$ & 29094 & $\alpha=0,11$ e $\beta=1$ \\
\hline Holt-Winters Aditivo & 12808 & $\begin{array}{c}\alpha=0,5 ; \beta=0,5 \\
\text { e } \quad \gamma=0,5\end{array}$ & 23526 & $\begin{array}{c}\alpha=0,5 ; \beta=0,5 \\
\text { e } \quad \gamma=0,5\end{array}$ \\
\hline $\begin{array}{l}\text { Holt-Winters Aditivo } \\
\text { Otimizado }\end{array}$ & 10351 & $\begin{array}{c}\alpha=0,34 ; \beta=0,03 \\
\text { e } \quad \gamma=0,40\end{array}$ & 11017 & $\begin{array}{l}\alpha=1 ; \beta=0 \\
\mathrm{e} \quad \gamma=0,19\end{array}$ \\
\hline Holt-Winters Multiplicativo & 25503 & $\begin{array}{c}\alpha=0,5 ; \beta=0,5 \\
\text { e } \quad \gamma=0,5\end{array}$ & 27108 & $\begin{array}{c}\alpha=0,5 ; \beta=0,5 \\
\text { e } \quad \gamma=0,5\end{array}$ \\
\hline $\begin{array}{l}\text { Holt-Winters Multiplicativo } \\
\text { Otimizado }\end{array}$ & 9908 & $\begin{array}{c}\alpha=0,26 ; \beta=0 \\
\text { e } \quad \gamma=1\end{array}$ & 17820 & $\begin{array}{c}\alpha=0,06 ; \beta=0 \\
\text { e } \quad \gamma=1\end{array}$ \\
\hline
\end{tabular}

Fonte: Autoria própria (2012)

Os resultados obtidos desse estudo preliminar, conforme Quadro 1, mostram um desempenho superior dos métodos Holt-Winters Sazonal Multiplicativo e o Aditivo da estatística MSE para as séries temporais dos produtos A e B, respectivamente. Assim, tais métodos devem ser preferidos aos demais métodos de suavização exponencial na previsão de valores futuros. 


\subsection{Modelagem e Previsão de Demanda}

A sistemática para estabelecer as previsões de demanda neste trabalho envolve a utilização de planilhas eletrônicas em ambiente MS-Excel® com o objetivo de selecionar o método de previsão de suavização mais apropriado, ou seja, o método capaz de prever acontecimentos futuros, tendo como propósito a redução do risco na tomada de decisão.

A modelagem para a previsão de demanda neste trabalho é apresentada apenas para os métodos de previsão de Holt-Winters escolhidos (menor MSE) conforme resultados do Quadro 1. Portanto, neste trabalho as previsões de demanda para os produtos A e B foram estabelecidas a partir desses métodos de previsão que melhor aderiram às séries temporais geradas a partir dos dados históricos de vendas. Um algoritmo, para o desenvolvimento dos métodos propostos neste trabalho inclui, basicamente, cinco etapas:

a) Calcular o nível básico $L_{t}$, para o período de tempo t, utilizando a equação 8 para o (Multiplicativo) e a equação 14 para o (Aditivo);

b) Calcular o valor da tendência estimada $T_{t}$, para o período de tempo t, utilizando a equação 9 para os casos (Multiplicativo e Aditivo);

c) Calcular o fator Sazonal estimado $S_{t}$, para o período de tempo t, utilizando equação 10 (Multiplicativo) e a equação 16 (Aditivo);

d) Calcular a previsão final $\hat{Z}_{t+n}$, para o período de tempo $\mathrm{t}+\mathrm{n}$, a partir da equação 7 (Multiplicativo e Aditivo);

e) Resolver o modelo matemático de programação não linear aplicando a ferramenta "Solver" do ambiente MS-Excel® para determinar os parâmetros $\alpha, \beta$ e $\gamma$ (constantes de suavização) que minimizam a função objetivo e assim determinar a previsão ótima, ou seja, resolver o modelo, minimizando o MSE, alterando $\alpha, \beta$ e $\gamma$, sujeito a $0 \leq \alpha, \beta, \gamma \leq 1$.

As Figuras 2 a 5 ilustram os gráficos das previsões obtidos pelos métodos Holt-Winters (Multiplicativo e Aditivo) e os dados reais para os produtos A e B. As Figuras 2 e 4 mostram a implementação desses métodos com constantes de suavização iguais a 0,5 para a série temporal do produto A e B, respectivamente. As Figuras 3 e 5 ilustram a solução ótima obtida com a alteração dos parâmetros (constantes de suavização) que minimizam o MSE com objetivo de otimizar a previsão a partir da resolução do modelo matemático de programação não linear. Desta forma, com base na análise gráfica das Figuras 3 e 5, verifica-se que as alterações das constantes de suavização produzem valores que tendem a aproximar-se dos dados originais. Assim, é possível obter-se um cenário de previsão que reproduz melhor as condições verificadas na série. 
Figura 2 - Planilha do método Holt-Winters Sazonal Multiplicativo para a previsão de demanda do produto A

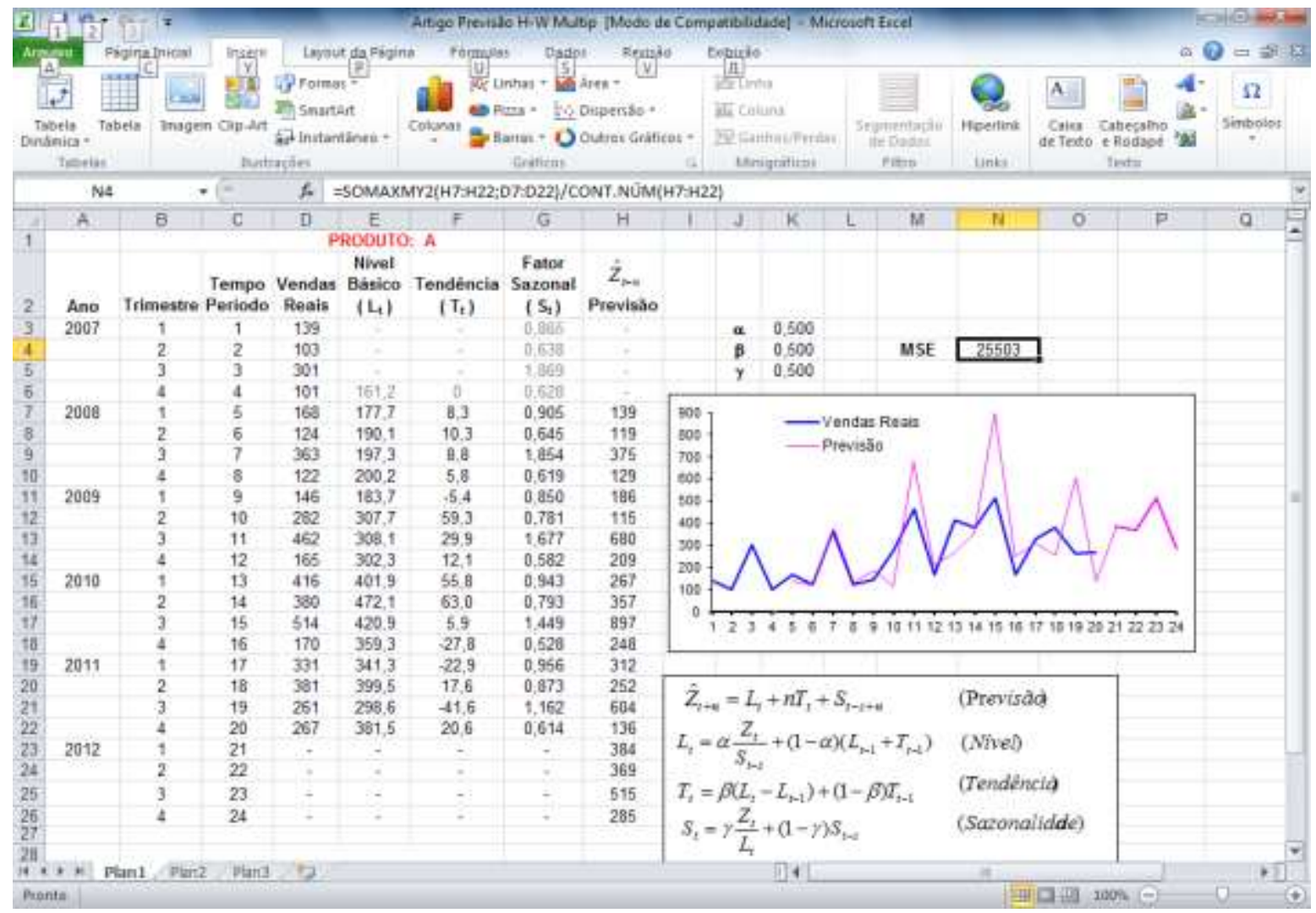

Fonte: Autoria própria (2012)

Figura 3 - Solução da previsão de demanda do produto A para o Método Holt-Winters Sazonal Multiplicativo

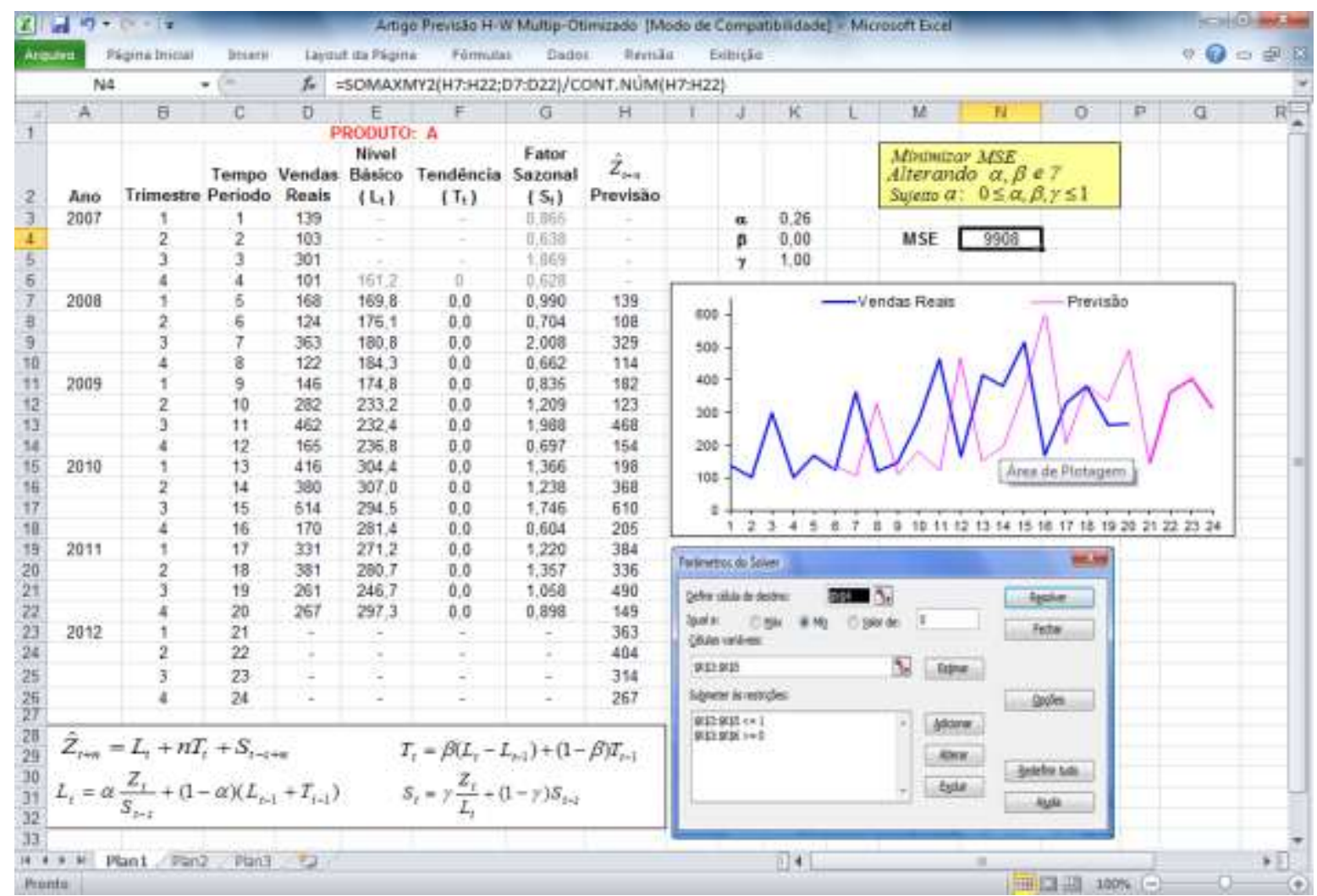

Fonte: Autoria própria (2012) 
Figura 4 - Planilha do método Holt-Winters Sazonal Aditivo para a previsão de demanda do produto B

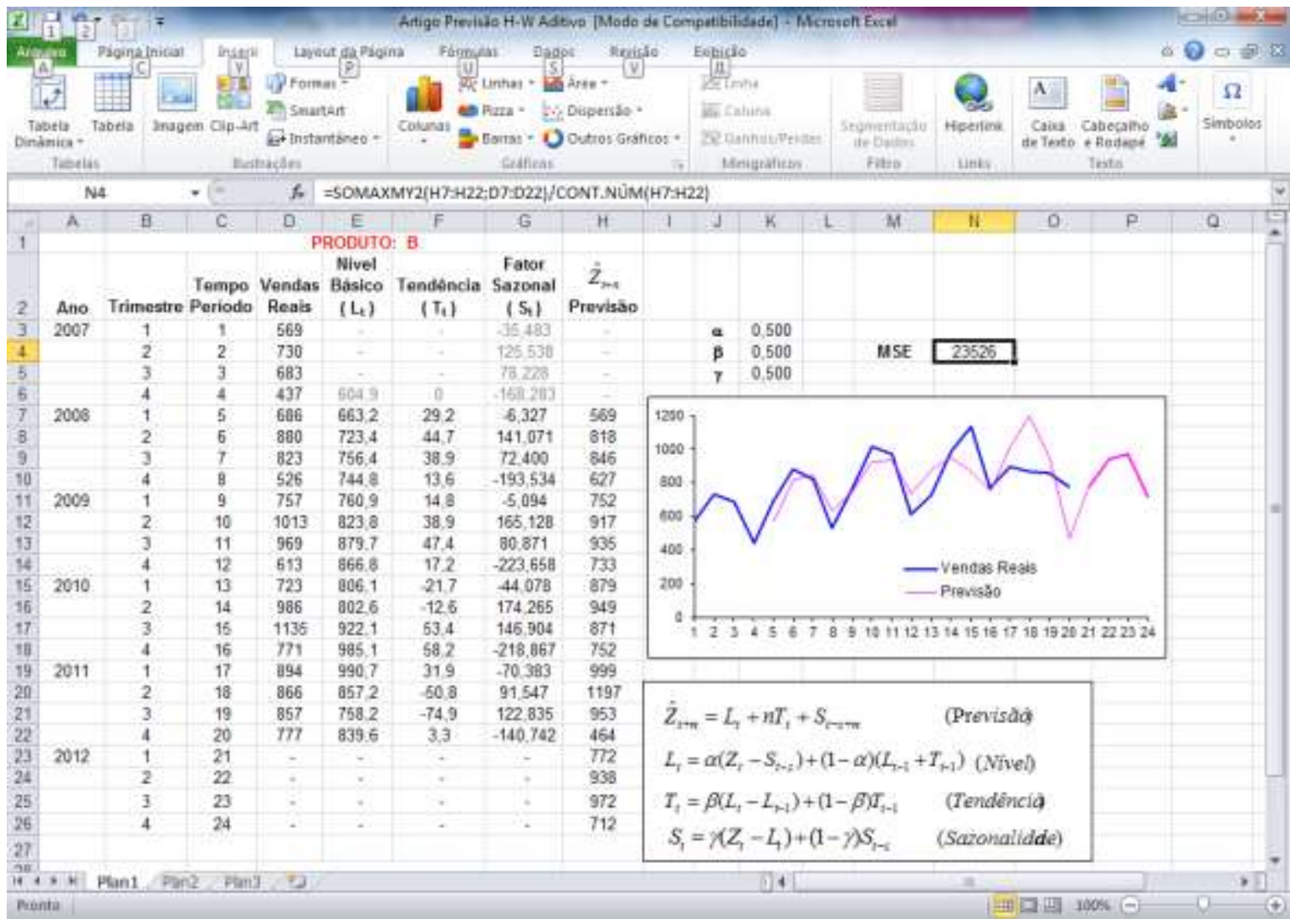

Fonte: Autoria própria (2012)

Figura 5 - Solução da previsão de demanda do produto B para o Método Holt-Winters Sazonal Aditivo

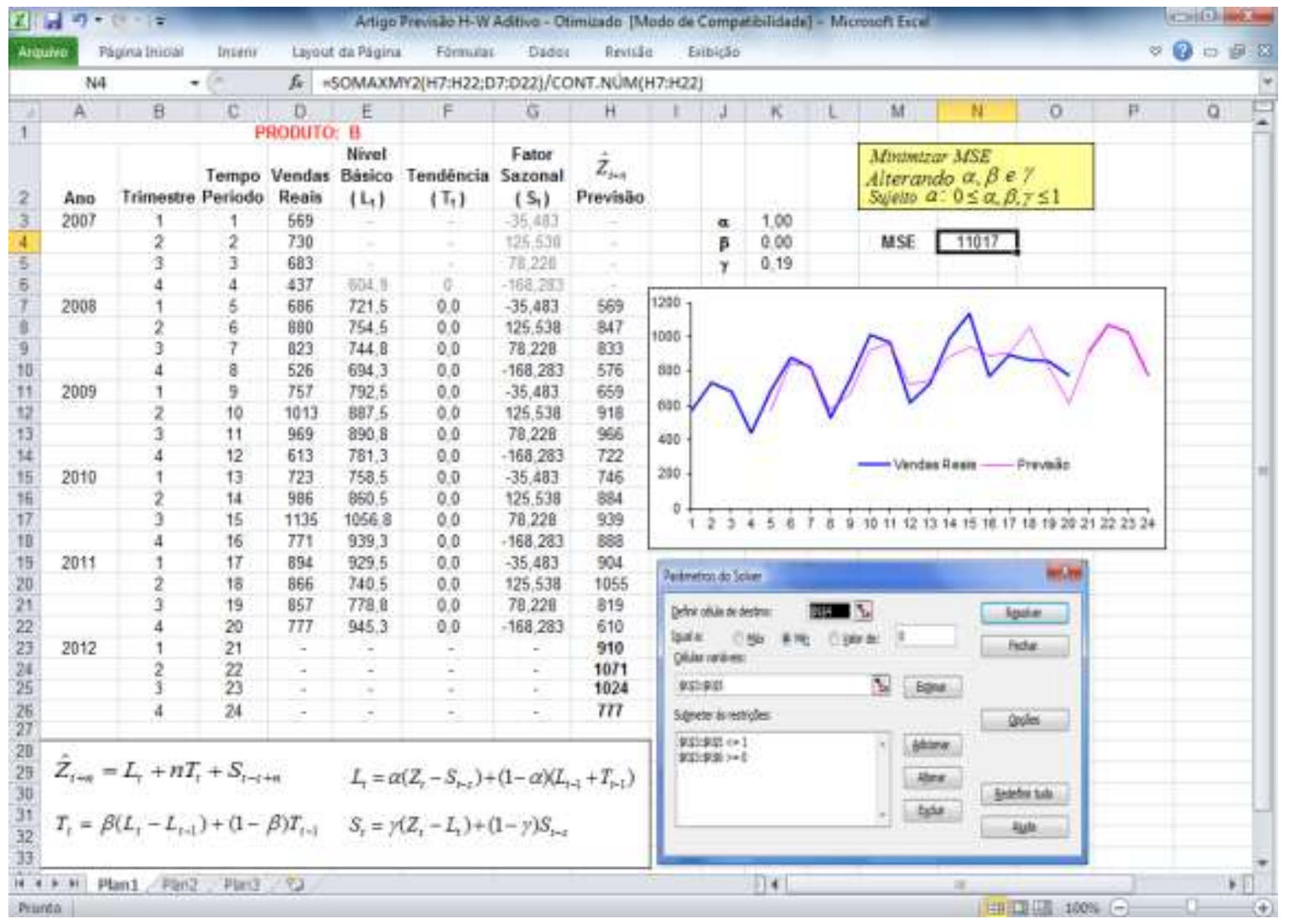

Fonte: Autoria própria (2012) 
Além disso, comparando os resultados do MSE entre os métodos de suavização estudados, os métodos Holt-Winters Sazonal Multiplicativo e Aditivo são selecionados pelo fato de ser capaz de produzirem uma função que melhor se adapta aos dados da série dos produtos A e B, respectivamente.

\section{Considerações Finais}

O objetivo principal desse trabalho foi testar a aplicação de métodos estatísticos de séries temporais para realizar prognósticos de variáveis, dentre os quais os modelos de suavização exponencial, em particular os métodos Holt-Winters que nos permite lidar com séries temporais que contém fatores tanto de tendência quanto de sazonalidade. O estudo mostrou a eficácia da aplicação de ambos os métodos produzindo resultados eficientes na previsão de demanda dos produtos analisados.

Nesse trabalho para estabelecer a previsão demanda dos dois produtos A e B que apresentam comportamento sazonal a essência da análise de séries temporais teve como objetivo minimizar os efeitos que os erros de previsões têm gerado a empresa considerada. A determinação de previsões de demanda adequadas e, ao mesmo tempo realistas, ou seja, com menor margem de erro a partir desses métodos constituiu-se de um grande diferencial para a empresa estudada que apresenta restrições de capacidade e flexibilidade limitada para responder mudanças em curto prazo devido a grande variedade de seus produtos. Para essa empresa essas previsões são utilizadas para programação de produção, planejamento da capacidade de produção, controle de estoques, aquisição de matéria prima e contratação de mão de obra.

Os resultados obtidos mostram que a implementação de métodos estatísticos de suavização exponencial Holt-Winters, como problemas não lineares, pode ser realizada eficientemente utilizando modelagem em planilhas eletrônicas em ambiente MS-Excel®, para selecionar o método mais apropriado e, sobretudo otimizar a previsão de demanda. Neste estudo, os modelos foram selecionados por apresentarem melhor MSE para previsão de demanda. É importante salientar ainda que a implementação de qualquer método de previsão pressupõe uma escolha adequada deste e de seus parâmetros, uma vez que a precisão da previsão de demanda depende em parte destas escolhas.

\footnotetext{
Abstract

The Holt-Winters exponential smoothing statistical methods are widely used for supplying shortterm forecasts on sales data and demand levels, due to its simplicity, low overhead, good precision, automatic adjustment capabilities, and rapid changes in temporal series analysis. The main objective of this study is to apply these statistical methods to achieve precise short-term forecasts on sales demand planning and based on this forecast demand analysis making decisions which seek to contemplate continual optimized management in the manufacturing processes in a company of metal mechanic sector of the northern region of Santa Catarina State. Our study proposes a temporal
} 
series sales analysis in two product categories, based on seasonal effects which exert a significant impact on the costs and investments of the company. The choice of the forecasting method for each one of these series must consider types of seasonal effects that establish the adequate utilization of the Holt-Winters Multiplicative Season as compared to the Additive Seasonal methods. The obtained results, as outlined in this study have been exclusively based on historical data on sales demands which have been fundamental for the forecast analysis of this company, paying special attention to the level of accuracy of utilized forecasting methods and the adoption of a forecast error monitoring system, an important aspect for making corrective action decisions, whenever deemed necessary.

Key-words: Statistical Methods, Time Series Analysis, Forecasting.

\section{Referências}

BERMÚDEZ, J. D.; SEGURA, J. V.; VERCHER, E. A decision support system methodology for forecasting of time series based on soft computing. Computational Statistics \& Data Analysis, v. 51, p.177-191, 2006.

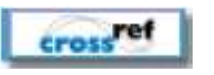

BILLAH, B.; KING, M. L.; SNYDER, R. D.; KOEHLE, A. B. (2006). Exponential smoothing model selection for forecasting. International Journal of Forecasting, v.22, n. 2, p. 239-247, 2006.

cross ref

BROWN, R.G. Statistical Forecasting for Inventory Control. McGraw-Hill, New York, NY, 1959.

BROWN, R. G. Smoothing, forecasting, and prediction. Englewood Cliffs, N.J.: Prentice Hall, 1963.

HENNING E.; ALVES C.C.; KONRATH, A.C. Previsão de vendas de rodízios para móveis em uma empresa de médio porte. In: ICPR AMERICAS - 5th Americas International Conference on Production Research, Bogotá, Colômbia, 2010. Proceedings... Bogotá: 2010.

GARDNER E.S. Exponential Smoothing: The State of the Art - Part II. International Journal of Forecasting, v.22, n.4, p. 637-666, 2006.

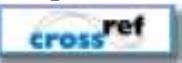

GARDNER E.S. Exponential Smoothing: The State of the Art. Journal of Forecasting, n.4, p. 1-28, 1985.

cross ${ }^{\text {ref }}$

GELPER, S.; FRIED, R ; CROUX, C. Robust forecasting with exponential and Holt-Winters smoothing. Journal of Forecasting, v.29, n. 3, p. 285-300, 2010.

HYNDMAN, R. J.; KOEHLER, A. B.; ORD, J. K.; SNYDER, R. D.; GROSE, S. A state space framework for automatic forecasting using exponential smoothing methods. International Journal of Forecasting, vol.18, n. 3, p. 439-454, 2002.

cross ref

HYNDMAN, R. J.; KOEHLER, A. B.; ORD, J. K. ; SNYDER, R. D. Forecasting with exponential smoothing: the state space approach, Springer-Verlag, Berlin, 2008.

cross ref

HOLT, C.E. Forecasting Trends and Seasonal by Exponentially Weighted Averages. ONR Memorandum No. 52, Carnegie Institute of Technology, Pittsburgh, USA, 1957.

KRAJEWSKI, L.; RITZMAN, L; MALHOTRA, M. Operations Management: Processes and Value Chain, Eight Edition, Pearson Prentice Hall, 2007. 
MAKRIDAKIS, S.; HIBON, M. The M3-competition: Results, conclusions and implications. International Journal of Forecasting, v.16, 451-476, 2000.

cross ref

MAKRIDAKIS, S.; WHEELWRIGHT, S.; HYNDMAN, R.J. Forecasting Methods and Applications. 3. ed. New York: John Wiley \& Sons, 1998.

MONTGOMERY, D.C.; JENNINGS, C.L.; KULAHCI, M. Introduction to Time Series Analysis and Forecasting. New Jersey: John Wiley \& Sons, 2008.

MOREtTIN, P. A.; TOLOI, C. M. Análise de Séries Temporais. 2ª ed. São Paulo: Editora Edgard Blucher, 2006.

PELLEGRINI, F.R.; FOLIATTO, F.S. Passos para Implantação de Sistemas de Previsão de Demanda -Técnicas e Estudo de Caso. Revista Produção, v. 11, n. 1, p. 43-64, 2001.

RAGSDALE, C.T. Modelagem e Análise de Decisão. São Paulo: Cengage Learning, 2009.

RASMUSSEN, R. On time series data and optimal parameters. Omega, v. 32, n. 2, p. 111-120, 2004.

cross ref

SOUZA, G.P.; SAMOHYL, R.W.; MIRANDA, R.G. Métodos Simplificados de Previsão Empresarial. Rio de Janeiro: Editora Ciência Moderna, 2008.

SOUZA, R.C. Modelos Estruturais para Previsão de Séries Temporais: Abordagens Clássica e Bayesiana, IMPA (17 Colóquio Brasileiro de Matemática), Rio de Janeiro, 1989.

WINTERS, P. R. Forecasting sales by exponentially weighted moving averages. Management Science, v. 6, n. 3, p. 324-342, 1960.

eross $r$ ef

\section{Dados dos autores:}

Nome Completo: Andrey Jonas Veríssimo

Filiação institucional: Universidade da Região de Joinville

Departamento: Departamento de Engenharia de Produção

Função ou cargo ocupado: Engenheiro de Produção Mecânica

Endereço: Rua Paulo Malschitzki, 10 Campus Universitário - Zona Industrial, Joinville/SC.

CEP: $89.219-710$

Telefone: (47) 3461-9066

e-mail: andrey-verissimo@bol.com.br

\section{Nome Completo: Custódio da Cunha Alves}

Filiação institucional: Universidade da Região de Joinville

Departamento: Departamento de Engenharia de Produção

Função ou cargo ocupado: Professor Titular

Endereço: Rua Paulo Malschitzki, 10 Campus Universitário - Zona Industrial, Joinville/SC.

CEP: $89.219-710$ 
Telefone: (47) 3461-9066

e-mail: custodio.alves@univille.br

\section{Nome Completo: Elisa Henning}

Filiação institucional: Centro de Ciências Tecnológicas / Universidade do Estado de Santa Catarina Departamento: Departamento de Matemática

Função ou cargo ocupado: Professor Adjunto

Endereço: Rua Paulo Malschitzki, s/n - Campus Universitário Prof. Avelino Marcante - Bairro Zona Industrial Norte - Joinville/SC.

CEP: 89.219-710

Telefone: (47) 40097978

e-mail: elisa.henning@udesc.br

\section{Nome Completo: Claiton Emilio Do Amaral}

Filiação institucional: Universidade da Região de Joinville

Departamento: Departamento de Engenharia de Produção

Função ou cargo ocupado: Coordenador do Curso de Engenharia de Produção Mecânica

Endereço: Rua Paulo Malschitzki, 10 Campus Universitário - Zona Industrial, Joinville/SC.

CEP: 89.219-710

Telefone: (47) 3461-9066

e-mail: claiton.emilio@gmail.com

\section{Nome Completo: Altair Carlos da Cruz}

Filiação institucional: Universidade da Região de Joinville

Departamento: Departamento de Engenharia de Produção

Função ou cargo ocupado: Chefe do Depto. de Engenharia de Produção Mecânica

Endereço: Rua Paulo Malschitzki, 10 Campus Universitário - Zona Industrial, Joinville/SC.

CEP: 89.219-710

Telefone: (47) 3461-9066

e-mail: altair.carlos@univille.br

Enviado em: 06/12/2012

Aprovado em: 22/01/2013 